\title{
Negative effective permeability of multilayers of ordered arrays of metal-dielectric nanosandwiches
}

\author{
C. Tserkezis, ${ }^{a}$ N. Stefanou, ${ }^{a}$ G. Gantzounis, ${ }^{a}$ and N. Papanikolaou ${ }^{b}$ \\ ${ }^{a}$ Section of Solid State Physics, University of Athens, Panepistimioupolis, GR-157 84 Athens, \\ Greece \\ ${ }^{b}$ Institute of Microelectronics, NCSR "Demokritos", Ag. Paraskevi, GR-153 10 Athens, Greece
}

\begin{abstract}
We present a thorough theoretical study of the optical properties of periodic structures built of silver and silica nanodisks in a sandwich-like configuration, by means of full electrodynamic calculations using the extended layer-multiple-scattering method. The strong coupling of the metallic nanoparticles and the resulting plasmon hybridization lead to collective electric and magnetic resonant modes, which can be tuned by changing the structural parameters, such as nanoparticle size and lattice constant. We analyze the response of single- and multi-layer architectures of ordered arrays of such nanosandwiches on a dielectric substrate to externally incident light and evaluate the corresponding effective permittivity and permeability functions. Our results reveal the existence of optical magnetism, with a strong negative effective permeability over a tunable spectral range at near-infrared and visible frequencies. We introduce the complex photonic band structure as a tool in the study of three-dimensional metamaterials and establish additional criteria for the validity of their effective-medium description. Our work demonstrates the efficiency of the recently developed extended layer-multiple-scattering method in the study of metamaterials of composite metal-dielectric particles of arbitrary shape.
\end{abstract}

Keywords: metal-dielectric nanosandwiches, plasmon hybridization, negative effective permeability, multiple scattering method, complex band structure

\section{INTRODUCTION}

Composite structures of coupled metallic nanoparticles exhibit a plethora of interesting phenomena that stem from the existence of strong and tunable plasmonic resonances, ${ }^{1}$ thus offering impressive opportunities for tailoring the light-matter interaction. Among the different architectures of such coupled particles, in-tandem pairs of metallic nanodisks separated by a dielectric spacer, so-called metal-dielectric-metal nanosandwiches, attract considerable attention for a variety of reasons, ${ }^{2-7}$ not least of which are potential applications as negative-index metamaterials at visible and near-infrared frequencies. ${ }^{8-10}$ When two metallic nanodisks are brought into strong coupling in a sandwich-like configuration, plasmon hybridization occurs and the initial degenerate plasmon modes split into two resonances: One is symmetric, i.e., the dipole moments of the nanodisks are parallel, and the other is antisymmetric, i.e., the dipole moments are in opposite directions. While the symmetric resonance has an electric dipolar character, the antisymmetric one generates a loop-like current in the nanodisk pair and thus a dipolar magnetic moment. An array of such "magnetic metamolecules" can exhibit a negative effective permeability, ${ }^{10}$ which is an essential ingredient in the design of negative-index metamaterials. Compared to pairs of rods or of cut-wires, the optical behavior of disk pairs is likely to be more isotropic, since the latter are invariant under rotation about their axis. In the last years, the optical response of metal-dielectric-metal nanosandwiches has been extensively investigated, supported by the broad availability of lithographic tools, which enable systematic studies of well-defined structures. ${ }^{2-10}$ In the present work, we report a thorough investigation of the effective optical properties of periodic multilayers of silver-silica-silver nanosandwiches by means of full electrodynamic calculations using the extended layer-multiple-scattering method. ${ }^{11-13}$ We analyze the results in conjunction with relevant extinction spectra and complex band structure diagrams, and emphasize aspects of the underlying physics which have not been discussed previously.

Further author information: (Send correspondence to C.T.) C.T.: E-mail: ctserk@phys.uoa.gr, Telephone: +30 2107276758

Metamaterials IV, edited by Vladimir Kuzmiak, Peter Markoš, Tomasz Szoplik, Proc. of SPIE Vol. 7353, 735305 - @ 2009 SPIE · CCC code: 0277-786X/09/\$18 · doi: 10.1117/12.820440

Proc. of SPIE Vol. 7353 735305-1 


\section{COUPLED PLASMON MODES IN A NANOSANDWICH}

Let us first consider a single metallic nanodisk, which is the building unit of a nanosandwich. This is characterized by a (relative) magnetic permeability $\mu_{\mathrm{s}}=1$ and a (relative) electric permittivity $\epsilon_{\mathrm{s}}$, which is a function of the angular frequency $\omega$. We assume, to begin with, that $\epsilon_{\mathrm{s}}$ has the simple yet effective Drude form ${ }^{14}$

$$
\epsilon_{\mathrm{S}}(\omega)=1-\frac{\omega_{\mathrm{p}}^{2}}{\omega\left(\omega+\mathrm{i} \tau^{-1}\right)},
$$

where $\omega_{\mathrm{p}}$ is the bulk plasma frequency and $\tau$ the relaxation time of the conduction-band electrons of the metal, which accounts for dissipative losses. When using the Drude permittivity of Eq. (1), it is convenient to express the frequency in units of $\omega_{\mathrm{p}}$ and consider $c / \omega_{\mathrm{p}}$ as the length unit, where $c$ is the velocity of light in vacuum. We note that, assuming a typical value of $10 \mathrm{eV}$ for $\hbar \omega_{\mathrm{p}}, c / \omega_{\mathrm{p}}$ corresponds to about $20 \mathrm{~nm}$.

The scattering properties of a single particle can be obtained through the so-called $T$ matrix, which is defined, in general terms, as the matrix that transforms the incident wave field into the scattered wave field. For particles of cylindrical symmetry, the $T$ matrix in the basis of vector spherical waves $|P \ell m\rangle$, where $\ell=1,2, \ldots$ and $m=-\ell,-\ell+1, \ldots, \ell$ are the usual angular-momentum indices, and $P=E, H$ denotes the electric and magnetic multipole modes, respectively, has a block-diagonal form. Its eigenvalues are characterized by a given value of $|m|$ and a given parity, i.e., the corresponding eigenfields are either even or odd upon inversion. In general, a single particle supports resonant modes of the electromagnetic (EM) field at the poles of the eigenvalues of the corresponding $T$ matrix in the lower complex frequency half-plane near the real axis. For example, a metallic nanodisk in a dielectric host medium exhibits resonances that correspond to collective electron oscillations at the surface of the particle and are termed particle-plasmon modes. Though, as stated above, the $T$ matrix is not diagonal in the $|P \ell m\rangle$ representation and thus there is no clear assignment of its eigenvalues according to $P$ and $\ell$, the particle-plasmon modes may have a predominant polarization and $2^{\ell}$-pole character.

In the left-hand diagram of Fig. 1 we show the scattering cross section, normalized to the geometric cross section, of a single metallic nanodisk in air, illuminated by a plane wave at an angle $\theta=45^{\circ}$ with respect to the disk axis (taken as the $z$ axis). The incident wave is $p$-polarized, i.e., the electric field oscillates in the plane of incidence, which is defined by the disk axis and the direction of incidence. The disk is described by the Drude permittivity given by Eq. (1) without dissipative losses $\left(\tau^{-1}=0\right)$, and has a radius $S=2.5 c / \omega_{\mathrm{p}}$ and a thickness $h=c / \omega_{\mathrm{p}}$. In this case, obviously, the absorption cross section vanishes. The excitation of several particle-plasmon modes is clearly visible in the figure. The peaks at $0.293 \omega_{\mathrm{p}}, 0.402 \omega_{\mathrm{p}}$, and $0.465 \omega_{\mathrm{p}}$ are associated with particle-plasmon modes corresponding to $m= \pm 1, m= \pm 2$, and $m= \pm 3$, respectively. All these modes are predominantly of electric type and have a mainly dipole, quadrupole, and octapole character, respectively.

In the right-hand diagram of Fig. 1 we show the normalized scattering and absorption cross sections of an actual silver nanodisk, of dimensions $S=50 \mathrm{~nm}, h=20 \mathrm{~nm}$, in air. Again, we assume a $p$-polarized plane wave, incident at an angle $\theta=45^{\circ}$. Now, for the dielectric function of silver we interpolate to the bulk values measured by Johnson and Christy ${ }^{15}$ that include dissipative losses. It can be seen from the figure that, in the frequency region which interests us here, only the lowest dipole-like resonance survives in the scattering cross section. The higher particle-plasmon modes, which have very long lifetimes compared to the absorption time, are fully absorbed and the absorption cross section is characterized by broad resonances about the eigenfrequencies of the particle-plasmon modes.

We note that, for the numerical evaluation of the elements of the $T$ matrix for the particles under consideration, we employed the extended-boundary-condition method, properly adapted. ${ }^{13}$ Truncating the relevant angular-momentum expansions at $\ell_{\max }=12, \ell_{\text {cut }}=16$ and using a Gaussian quadrature integration formula with 4000 points for the integrals involved, our results have a relative error less than $10^{-3}$ in the considered range of frequencies.

In a nanosandwich configuration, the dipolar plasmon modes of the individual metallic nanodisks interact with each other and give rise to a symmetric and an antisymmetric resonant optical mode, by analogy to the formation of bonding and antibonding electron orbitals in diatomic molecules. In this respect, metal-dielectricmetal nanosandwiches constitute a new class of photonic metamolecules. In their symmetric optical mode, the electron gas oscillates in phase in the two metallic nanodisks. In the antisymmetric one, these oscillations have 

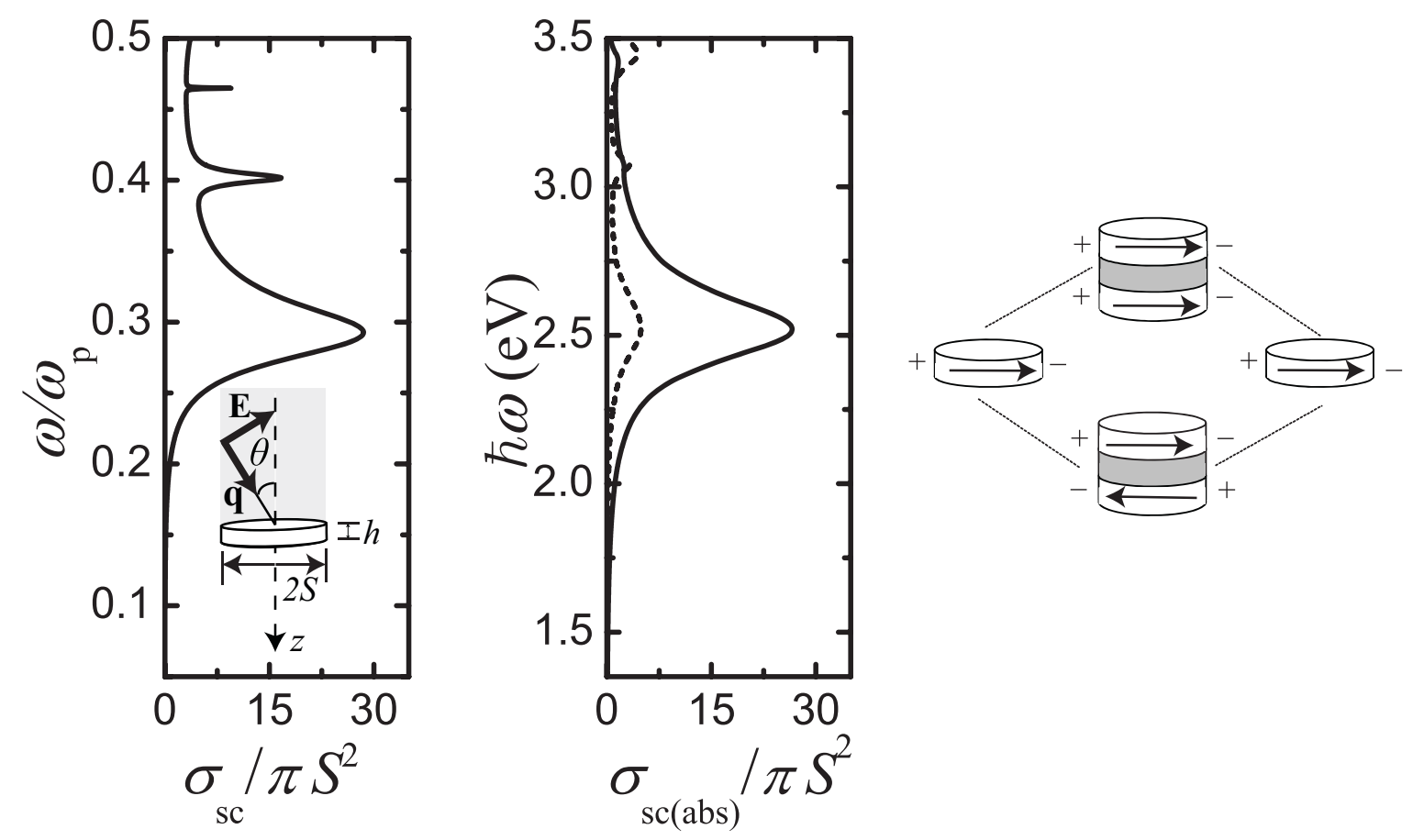

Figure 1. The scattering (solid lines) and absorption (dashed lines) cross sections of a silver nanodisk in air, for $p$-polarized light incident at an angle $\theta=45^{\circ}$ with respect to the disk axis, as shown in the inset. Left-hand diagram: The disk has a radius $S=2.5 c / \omega_{\mathrm{p}}$ and a thickness $h=c / \omega_{\mathrm{p}}$; its permittivity is given by Eq. (1) with $\tau^{-1}=0$, which implies that the absorption cross section is identically zero. Right-hand diagram: The experimental permittivity of bulk silver, measured by Johnson and Christy, ${ }^{15}$ is used for a disk of dimensions $S=50 \mathrm{~nm}$ and $h=20$ nm. Plasmon hybridization in a metal-dielectric-metal nanosandwich is shown schematically in the margin.

opposite phase, leading effectively to an electric current nanoloop that corresponds to a magnetic-type resonance. It is worthnoting that, contrary to the formation of bonding and antibonding orbitals in diatomic molecules, in a metal-dielectric-metal nanosandwich the low-frequency hybrid plasmonic mode is antisymmetric and the high-frequency one is symmetric. This apparently counter-intuitive situation can be understood as follows. Charge oscillations associated with an electric-dipole plasmon mode in a single metallic nanodisk are sustained by restoring forces acting on the collectively displaced conduction-band electrons. In an in-tandem pair of such nanodisks, charge distribution leads to reduction of the appearing restoring forces in the configuration of the antisymmetric mode and enhancement in the case of the symmetric mode. Consequently, the eigenfrequency of the antisymmetric mode is lowered and that of the symmetric mode is raised (see margin of Fig. 1). The situation is reversed if the two nanodisks are on the same plane.

\section{S-MATRIX METHOD FOR LAYERED STRUCTURES}

We assume, to begin with, a plane of nonoverlapping particles, at $z=0$, centered on the sites $\mathbf{R}_{n}$ of a given two-dimensional (2D) lattice. Let a plane EM wave be incident on this layer. Because of the 2D periodicity of the structure under consideration, we write the component of the wave vector of the incident wave parallel to the layer, $\mathbf{q}_{\|}$, as $\mathbf{q}_{\|}=\mathbf{k}_{\|}+\mathbf{g}^{\prime}$ where $\mathbf{k}_{\|}$, the reduced wave vector in the surface Brillouin zone (SBZ), is a conserved quantity in the scattering process and $\mathbf{g}^{\prime}$ is a certain reciprocal vector of the given lattice. Therefore, the wave vector of the incident wave has the form $\mathbf{K}_{\mathbf{g}^{\prime}}^{ \pm}=\mathbf{k}_{\|}+\mathbf{g}^{\prime} \pm\left[q^{2}-\left(\mathbf{k}_{\|}+\mathbf{g}^{\prime}\right)^{2}\right]^{1 / 2} \widehat{\mathbf{e}}_{z}$, where $\widehat{\mathbf{e}}_{z}$ is the unit vector along the $z$ axis and the + or - sign refers to incidence from the left $(z<0)$ or from the right $(z>0)$, i.e., a wave propagating towards the $z>0$ or the $z<0$ half space, respectively. Since $\omega$ and $\mathbf{k}_{\|}$are conserved quantities in the scattering process, the scattered field will consist of a series of plane waves with wave vectors

$$
\mathbf{K}_{\mathbf{g}}^{ \pm}=\mathbf{k}_{\|}+\mathbf{g} \pm\left[q^{2}-\left(\mathbf{k}_{\|}+\mathbf{g}\right)^{2}\right]^{1 / 2} \widehat{\mathbf{e}}_{z}, \forall \mathbf{g}
$$


and polarizations along $\widehat{\mathbf{e}}_{1}$ and $\widehat{\mathbf{e}}_{2}$ (polar and azimuthal unit vectors, respectively, associated with every $\mathbf{K}_{\mathrm{g}}^{s}$, $s= \pm$ ). Though the scattered wave consists, in general, of a number of diffracted beams corresponding to different 2D reciprocal-lattice vectors $\mathbf{g}$, only beams for which $K_{\mathbf{g} z}^{s}$ is real constitute propagating waves. When $\left(\mathbf{k}_{\|}+\mathbf{g}\right)^{2}>q^{2}$ the corresponding wave decays to the right for $s=+$, and to the left for $s=-$; and the corresponding unit vectors $\widehat{\mathbf{e}}_{\mathrm{p}}$ become complex but they are still orthonormal $\left(\widehat{\mathbf{e}}_{\mathrm{p}} \cdot \widehat{\mathbf{e}}_{\mathrm{p}^{\prime}}=\delta_{\mathrm{pp}^{\prime}}, \mathrm{p}, \mathrm{p}^{\prime}=1,2\right)$.

The scattering $S$ matrix transforms the incident into the outgoing wave field (with all plane-wave components expanded about a common origin of coordinates). Explicit expressions for the matrix elements $S_{\mathbf{g p} ; \mathbf{g}^{\prime} \mathbf{p}^{\prime}}$ are summarized elsewhere. ${ }^{11,12}$ One can use the same notation (the calculation is of course much easier) to describe scattering by homogeneous layers: a homogeneous plate, or an interface between two homogeneous media. In these cases the $S$ matrix is diagonal in $\mathbf{g}$ because of the translation invariance parallel to the $x y$ plane. The $S$ matrix of multilayers with the same $2 \mathrm{D}$ periodicity is obtained by combining the corresponding matrices of the component layers, with appropriate phase factors, in order to describe multiple-scattering between the layers to any order. For a three-dimensional (3D) crystal consisting of an infinite periodic sequence of layers, such as those described here above, along the $z$ direction, applying the Bloch condition for the wave field in the region between two consecutive unit slabs leads to an eigenvalue equation, which gives the $z$ component of the Bloch wave vector $k_{z}$, for given $\omega$ and $\mathbf{k}_{\|}{ }^{11,12}$

\section{MULTILAYERS OF PERIODIC ARRAYS OF NANOSANDWICHES}

We now apply the extended layer-multiple-scattering method ${ }^{11-13}$ to study the effective optical response of layered structures of silver-silica-silver nanosandwiches, arranged on a hexagonal lattice determined by the primitive vectors $\mathbf{a}_{1}=a_{0}(1,0,0), \mathbf{a}_{2}=a_{0}(1 / 2, \sqrt{3} / 2,0)$, on a quartz substrate $\left(\epsilon_{\text {quartz }}=2.13, \mu_{\text {quartz }}=1\right)$. A periodic array of nanosandwiches is built of three consecutive layers of appropriate nanodisks, through the proper combination of the $S$ matrices of the component layers. ${ }^{11,12}$ For the permittivity of silver we interpolate to the bulk values measured by Johnson and Christy ${ }^{15}$ while for silica we assume a permittivity of 2.13 . We consider nanosandwiches of radius $S=50 \mathrm{~nm}$, typical thickness $h_{1}=20 \mathrm{~nm}$ silver, $h_{2}=40 \mathrm{~nm}$ silica, $h_{3}=20 \mathrm{~nm}$ silver, and a lattice constant $a_{0}=200 \mathrm{~nm}$. In order to ensure adequate convergence in our calculations, we truncate the spherical-wave expansions at $\ell_{\max }=15$ and take into account 151 reciprocal-lattice vectors in the relevant plane-wave expansions, while the scattering $T$ matrix of the single nanodisk is calculated with $\ell_{\text {cut }}=20$ and a Gaussian quadrature integration formula with 6000 points. ${ }^{13}$

Hybridization between the particle-plasmon modes of the two metallic nanodisks of a single nanosandwich can be studied by keeping their thickness fixed $\left(h_{1}=h_{3}\right)$ and varying the thickness $h_{2}$ of the dielectric spacer, in a relatively sparse array of nanosandwiches. We have shown that, ${ }^{10}$ for very thin dielectric spacers, the extinction spectrum (extinction=negative logarithm of the transmittance) tends to a single-peak structure, which is essentially the particle-plasmon resonance in the corresponding array of homogeneous silver disks of double thickness. When the separation between silver disks in the nanosandwich is larger, the spectra exhibit two distinct peaks that originate from the excitation of the composite plasmon modes of the individual nanosandwiches, weakly interacting between them. With increasing thickness of the dielectric spacer, the high-frequency peak shifts to the red while the low-frequency peak shifts to the blue and gradually becomes more pronounced. It is worthnoting that the shift of the low-frequency antisymmetric mode is larger than that of the high-frequency symmetric mode. Our results are in good agreement with existing experimental data on isolated gold-silica-gold nanosandwiches. ${ }^{2,4}$ The interaction between nanosandwiches increases as we reduce the lattice constant and is manifested as a small shift of the resonance peaks and more pronounced extinction. ${ }^{10}$

The effective electromagnetic EM parameters of an inhomogeneous metamaterial, i.e., the permittivity and permeability functions of an equivalent homogeneous medium, $\epsilon_{\mathrm{eff}}$ and $\mu_{\mathrm{eff}}$, respectively, are usually determined from the scattered field in the far zone by a finite slab of the metamaterial, of thickness $D$, under plane wave illumination. For this purpose, we invert the standard Fresnel equations which give the transmission and reflection coefficients of a homogeneous slab described by a refractive index $n_{2}$ and an impedance $z_{2}$, placed between two semi-infinite homogeneous media with $n_{1}, z_{1}$ and $n_{3}, z_{3} \cdot{ }^{10,16,17}$ Obviously, this procedure takes also into account the presence of the substrate. The effective permittivity and permeability of the slab are given by $\epsilon_{\text {eff }}=n_{2} / z_{2}$ and $\mu_{\text {eff }}=n_{2} z_{2}$. 


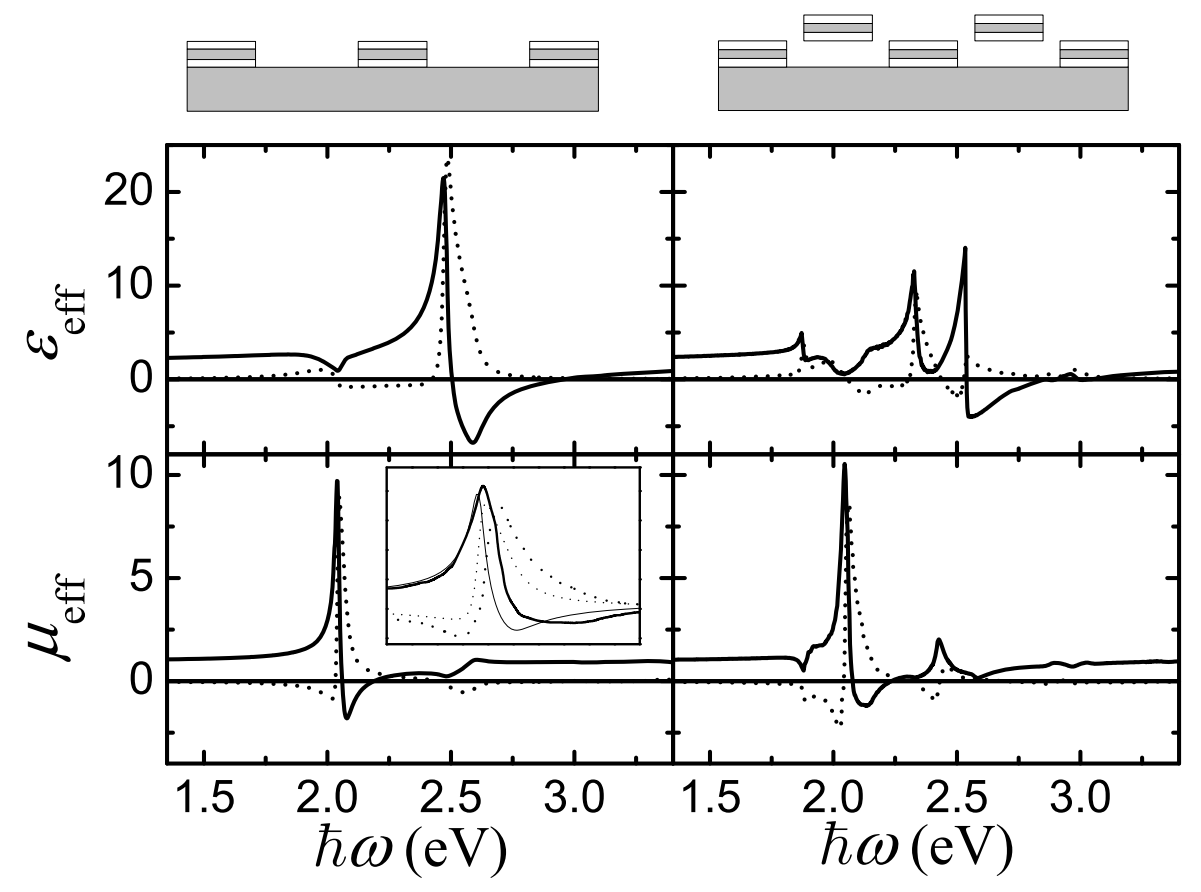

Figure 2. Real (solid lines) and imaginary (dotted lines) parts of the effective permittivity, $\epsilon_{\mathrm{eff}}$, and permeability, $\mu_{\mathrm{eff}}$, of one (left-hand panel) or two (right-hand panel) hexagonal arrays, with lattice constant $a_{0}=200 \mathrm{~nm}$, of silver-silica-silver nanosandwiches, with $S=50 \mathrm{~nm}, h_{1}=h_{3}=20 \mathrm{~nm}$, and $h_{2}=40 \mathrm{~nm}$, on a quartz substrate. In the bilayer case, the top layer is displaced by $\mathbf{a}_{3}=\left(a_{0} / 2, a_{0} \sqrt{3} / 6, h_{1}+h_{2}+h_{3}\right)$ with respect to the bottom layer. A detailed view of the effective permeability for one (thin lines) and two (thick lines) layers in the frequency region of the fundamental magnetic resonance is shown in the inset to the left bottom diagram.

The effective parameters $\epsilon_{\text {eff }}$ and $\mu_{\text {eff }}$, retrieved by the method described above at normal incidence for the monolayer of nanosandwiches described in the beginning of this section, are shown in the left-hand panel of Fig. 2. The effective thickness, $D$, of the homogeneous slab is taken equal to the total thickness of the nanosandwich, $h=h_{1}+h_{2}+h_{3}$, i.e., $D=80 \mathrm{~nm}$, though other choices for $D(>80 \mathrm{~nm})$ do not alter much our results. We obtain a pronounced resonance in the effective permeability in the frequency region of the antisymmetric mode and a similar resonance in the effective permittivity in the frequency region of the symmetric mode. It is worthnoting that the resonance in the permittivity is accompanied by a small resonance structure in the permeability, and vice versa. The effective permeability takes negative values as large as -1.77 between $2.05 \mathrm{eV}$ and $2.18 \mathrm{eV}$, while the effective permittivity becomes negative in the region from $2.50 \mathrm{eV}$ to $2.97 \mathrm{eV}$. However, these negative values are accompanied by relatively high losses, with the imaginary part of the effective refractive index being as high as 2.5. As we reduce the lattice constant, the interaction between nanosandwiches is stronger, resulting to more pronounced resonance structures in the extinction spectrum and more negative $\operatorname{Re} \mu_{\mathrm{eff}}$ within a broader frequency region. ${ }^{10}$

We next add, just on top of the given layer of nanosandwiches, a second similar layer, laterally displaced by $\left(\mathbf{a}_{1}+\mathbf{a}_{2}\right) / 3$ with respect to the bottom layer, i.e., the nanosandwiches of the second layer are placed at centers and those of the first layer at vertices of the triangles of the underlying $2 \mathrm{D}$ hexagonal lattice. The retrieved effective parameters $\epsilon_{\text {eff }}$ and $\mu_{\text {eff }}$ for this bilayer of nanosandwiches $(D=160 \mathrm{~nm})$ are shown in the right-hand panel of Fig. 2. In this case, strong interlayer coupling results in additional collective plasmonic resonances, which manifest themselves in the effective permittivity and permeability functions. However, as can be seen in the inset to the left bottom diagram of Fig. 2, the fundamental magnetic resonance and the associated negative effective permeability region remain quite robust and, as it turns out, they are also relatively insensitive to the presence of the substrate. We note that the interlayer interaction and the resulting coupled plasmonic resonances depend on the relative position of the two layers, as we verified by sliding the top layer at different positions. 
In both above cases (monolayer and bilayer), in the frequency region under consideration, the wavelength in the two semi-infinite embedding media (air and silica) is much larger than the in-plane period of the structure. Therefore, there is only a single propagating mode of the scattered EM field, for $\mathbf{g}=\mathbf{0}$ (see Eq. (2)), corresponding to outgoing waves in the substrate (refracted beam) and in air (reflected beam). All other components of the wave field (diffracted beams) are evanescent, as required in order for a description of a metamaterial with effective permittivity and permeability functions to be meaningful. However, though restriction to the $\mathbf{g}=\mathbf{0}$ block of the $S$ matrix is sufficient to obtain the scattered field by a finite slab of the given metamaterial in the far zone, the full $S$ matrix needs to be considered in order to describe interlayer coupling, which takes place through the near field in a multilayer slab. We note that, in the direction perpendicular to the layer(s), the period is also much shorter than the wavelength.

It should be pointed out that the definition of the effective parameters is based on the idea that a hypothetical homogeneous material slab mimics the inhomogeneous metamaterial in the sense that both systems produce the same outgoing field in the far zone (here for normal incidence). This does not mean that $\epsilon_{\mathrm{eff}}$ and $\mu_{\mathrm{eff}}$ describe the wave field inside the actual metamaterial where, at a given frequency, it has the form of a Bloch wave rather than a simple plane wave. However, the effective parameters must be such that these two waves obey the same dispersion relation and, therefore, have the same velocity. This remark is of course meaningful only if there is a single dominant relevant Bloch mode at the given frequency.

\section{THREE-DIMENSIONAL CRYSTALS OF NANOSANDWICHES}

We now build an infinite $3 \mathrm{D}$ crystal as a sequence of hexagonal arrays, with primitive vectors $\mathbf{a}_{1}=a_{0}(1,0,0)$ and $\mathbf{a}_{2}=a_{0}(1 / 2, \sqrt{3} / 2,0)$, of metal-dielectric-metal nanosandwiches. We assume that the permittivity of the metallic material is described by Eq. (1), and we deliberately disregard absorption $\left(\tau^{-1}=0\right)$ in order to be able to calculate the frequency band structure in an unambiguous manner. The nanosandwiches consist of two metallic nanodisks, of radius $S=2.5 c / \omega_{\mathrm{p}}$ and thickness $h_{1}=h_{3}=c / \omega_{\mathrm{p}}$, separated by a silica spacer of thickness $h_{2}=2 c / \omega_{\mathrm{p}}$. Therefore, $h=h_{1}+h_{2}+h_{3}=4 c / \omega_{\mathrm{p}}$ is the total thickness of the nanosandwich. The stacking sequence is defined by the primitive vector $\mathbf{a}_{3}=\left(a_{0} / 2, a_{0} \sqrt{3} / 6, h\right)$, so that the distance between successive lattice planes along the growth direction (the $z$ direction) is $d=h$, and we take $a_{0}=10 c / \omega_{\mathrm{p}}$.

Fig. 3 displays the complex photonic band structure of this crystal along the growth direction. At low frequencies we obtain a doubly degenerate linear dispersion curve, as expected for propagation in a homogeneous medium characterized by a frequency-independent effective refractive index. This band interacts with a narrow doubly degenerate band which originates from the antisymmetric plasmon modes of the individual nanosandwiches, weakly interacting between them. As a result, a frequency gap opens up about the crossing point of these bands, and is referred to as hybridization gap by analogy to the $s-d$ hybridization gap in the electron band structure of transition metals. ${ }^{14}$ In the gap region there are no propagating modes of the EM field and the real-frequency dispersion lines continue analytically in the complex $k_{z}$ plane. ${ }^{18}$ In principle, there is an infinite number of such complex bands but, over a gap region, it is the complex band of the appropriate symmetry (doubly degenerate in our case) with the smallest in magnitude imaginary part of $k_{z}$ which determines the transmission of EM waves through a finite slab of the crystal, along the given direction. In Fig. 3, near the gap edges, we show the real-frequency lines for complex eigenvalues $k_{z}$ that correspond to the doubly degenerate bands with the smallest in magnitude imaginary part (plotted in the grey-shaded areas). It can be seen that the relevant complex band near the top of the gap, at the Brillouin zone center, has Re $k_{z}=0$ and that near the bottom of the gap, at the Brillouin zone edge, has $\operatorname{Re} k_{z}=\pi / d$. It is interesting to examine whether such a complex band diagram can be associated to a homogeneous effective medium, at wavelengths long enough compared to the size of the particles and the lattice spacing. The presence of collective magnetic resonances in the crystal under consideration implies a resonant behavior of the effective permittivity function. Since there are no dissipative losses, we expect that $\mu_{\text {eff }}$ is real and exhibits an asymptotic variation taking negative values within a frequency interval next to the asymptote. In this region, assuming a real and positive $\epsilon_{\text {eff }}$, there can be

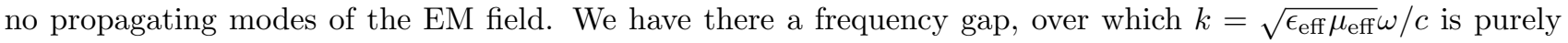
imaginary $(\operatorname{Re} k=0)$. Looking at the corresponding band structure of the actual crystal (Fig. 3), we see that the dominant evanescent modes near the top of the gap correspond, indeed, to Re $k_{z}=0$. Consequently, the form of the associated Bloch envelope wavefunctions $\left(\exp \left[-\operatorname{Im} k_{z} z\right]\right)$ is compatible with that of the evanescent modes 


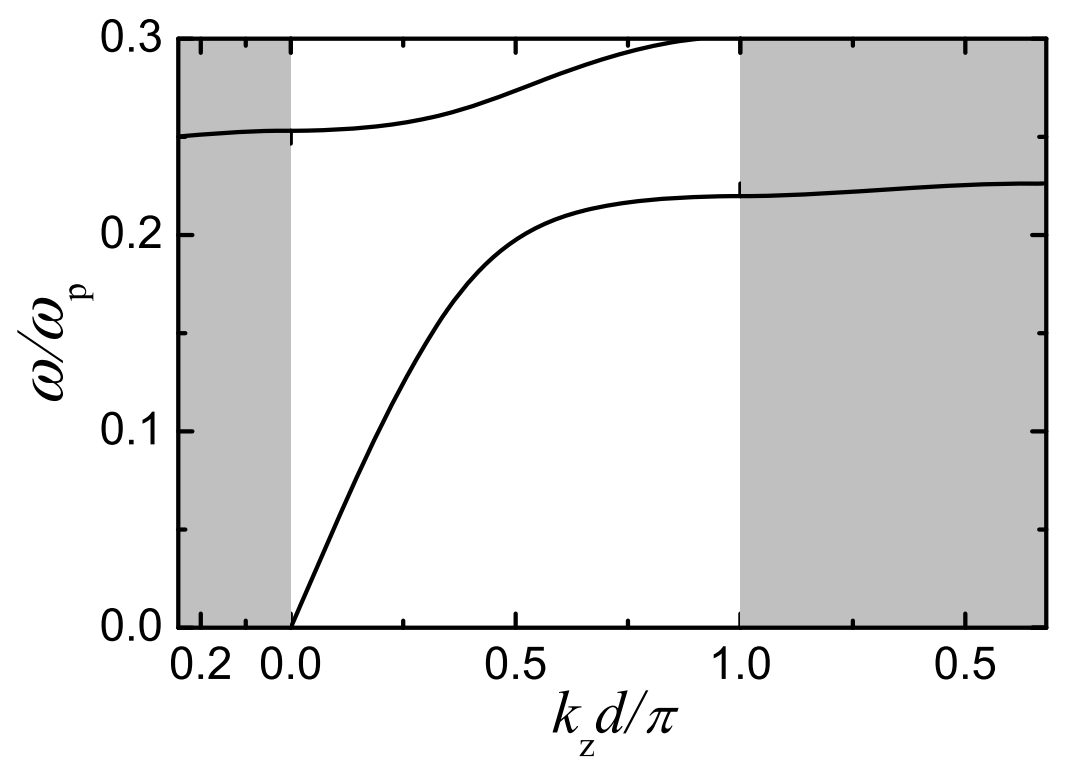

Figure 3. The photonic band structure of the 3D crystal described in the text, along the growth direction. Apart from the ordinary frequency bands (real $k_{z}$ ), we also show, near the gap edges, the doubly degenerate real-frequency lines for the complex eigenvalues $k_{z}$ with the smallest in magnitude imaginary part $\left(\operatorname{Im} k_{z}\right.$ is plotted in the gray-shaded areas).

supported in the effective medium, in the frequency region of negative $\epsilon_{\mathrm{eff}} \mu_{\mathrm{eff}}$. On the contrary, everywhere else in the gap, the dominant evanescent modes, resulting from destructive wave interference through multiple scattering in the periodic array of scatterers, correspond to $\operatorname{Re} k_{z} \neq 0$, and such waves cannot be formed in the effective medium. It becomes clear from the above that a negative-permeability (or negative-permittivity) 3D metamaterial should be associated with the existence of a gap region where the proper real-frequency line with the smallest $\left|\operatorname{Im} k_{z}\right|$ has $\operatorname{Re} k_{z}=0$. However, the $S$-matrix retrieval method leads, in general, to complex $\epsilon_{\text {eff }}$ and $\mu_{\text {eff }}$, even for systems made of non-absorptive components, especially in the frequency region of a resonance. Of course, in the absence of dissipation, the retrieval procedure itself ensures that the (complex) values of $\epsilon_{\text {eff }}$ and $\mu_{\text {eff }}$ are such that the absorption vanishes at any frequency. This can be achieved, e.g., through some fictitious dielectric gain $\left(\operatorname{Im} \epsilon_{\text {eff }}<0\right)$ which counterbalances fictitious magnetic $\operatorname{losses}\left(\operatorname{Im} \mu_{\text {eff }}>0\right)$, and vice versa. Obviously, this occurs for given slab thickness, specific characteristics of the incident fields, etc. and, therefore, these

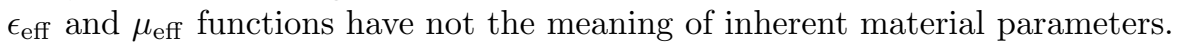

\section{CONCLUSION}

In summary, we reported on the effective optical properties of 2D and 3D ordered arrays of metal-dielectricmetal nanosandwiches on a dielectric substrate, by means of full electrodynamic calculations using the extended layer-multiple-scattering method. Recent metamaterial designs at visible and near-infrared frequencies focus on the possibility of generating magnetic dipole resonances from virtual current loops in various types of hybridized nanoplasmonic architectures. Periodic assemblies of nanosandwiches, such as those studied here, appear to be a promising route in this perspective, as they exhibit a strong and tunable magnetic response, are polarization insensitive at normal incidence, and can be easily fabricated. We analyzed a specific design of layered periodic structure of silver-silica-silver nanosandwiches, which exhibits a negative effective permeability over a tunable spectral range, and examined the variation of its resonant magnetic response as successive layers are stacked together to build a 3D crystal. We introduced the complex photonic band structure as a tool in the study of 3D metamaterials and established additional criteria for the validity of their effective-medium description. The present work demonstrates the efficiency of our recently developed extended layer-multiple-scattering method in the study of 2D and 3D metamaterials of composite metallodielectric nanoparticles of arbitrary shape. 


\section{ACKNOWLEDGMENTS}

This work was supported by the research program "Kapodistrias" of the University of Athens.

\section{REFERENCES}

1. Nordlander, P., Oubre, C., Prodan, E., Li, K. and Stockman, M. I., "Plasmon hybridization in nanoparticle dimers," Nano Lett. 4(5), 899-903 (2004).

2. Su, K. H., Wei, Q. H. and Zhang, X., "Tunable and augmented plasmon resonances of $\mathrm{Au} / \mathrm{SiO}_{2} / \mathrm{Au}$ nanodisks," Appl. Phys. Lett. 88(6), art. no. 063118, 1-3 (2006).

3. Pakizeh, T., Abrishamian, M. S., Granpayeh, N., Dmitriev, A. and Käll, M., "Magnetic-field enhancement in gold nanosandwiches," Opt. Express 14(18), 8240-8246 (2006).

4. Dmitriev, A., Pakizeh, T., Käll, M. and Sutherland, D. S., "Gold-silica-gold nanosandwiches: Tunable bimodal plasmonic resonators," Small 3(2), 294-299 (2007).

5. Wang, S.M., Li, T., Liu, H., Wang, F. M., Zhu, S. N. and Zhang, X., "Magnetic plasmon modes in periodic chains of nanosandwiches," Opt. Express 16(6), 3560-3565 (2008).

6. Pakizeh, T., Dmitriev, A., Abrishamian, M. S., Granpayeh, N. and Käll, M., "Structural asymmetry and induced optical magnetism in plasmonic nanosandwiches," J. Opt. Soc. Am. B 25(4), 659-667 (2008).

7. Ekinci, Y., Christ, A., Agio, M., Martin, O. J. F., Solak, H. H. and Löffler, J. F., "Electric and magnetic resonances in arrays of coupled gold nanoparticle in-tandem pairs," Opt. Express 16(17), 13287-13295 (2008).

8. Feth, N., Enkrich, C., Wegener, M. and Linden, S., "Large-area magnetic metamaterials via compact interference lithography," Opt. Express 15(2), 501-507 (2007).

9. Yuan, H. K., Chettiar, U. K., Cai, W., Kildishev, A. V., Boltasseva, A., Drachev, V. P. and Shalaev, V. M., "A negative permeability material at red light," Opt. Express 15(3), 1076-1083 (2007).

10. Tserkezis, C., Papanikolaou, N., Gantzounis, G. and Stefanou, N., "Understanding artificial optical magnetism of periodic metal-dielectric-metal layered srtuctures," Phys. Rev. B 78(16), art. no. 165114, 1-7 (2008).

11. Stefanou, N., Yannopapas, V. and Modinos, A., "Heterostructures of photonic crystals: Frequency bands and transmission coefficients," Comput. Phys. Commun. 113(1), 49-77 (1998).

12. Stefanou, N., Yannopapas, V. and Modinos, A., "MULTEM 2: A new version of the program for transmission and band structure calculations of photonic crystals," Comput. Phys. Commun. 132(1-2), 189-196 (2000).

13. Gantzounis, G. and Stefanou, N., "Layer-multiple-scattering method for photonic crystals of nonspherical particles," Phys. Rev. B 73(3), art. no. 035115, 1-10 (2006).

14. Ashcroft, N. W. and Mermin, N. D., [Solid State Physics], Saunders, New York (1976).

15. Johnson, P. B. and Christy, R. W., "Optical constants of the noble metals," Phys. Rev. B 6(12), 4370-4379 (1972).

16. Smith, D. R., Schultz, S., Markoš, P. and Soukoulis, C. M., "Determination of effective permittivity and permeability of metamaterials from reflection and transmission coefficients," Phys. Rev. B 65(19), art. no. 195104, 1-5 (2002).

17. Menzel, C., Rockstuhl, C., Paul, T., Lederer, F. and Pertsch, T., "Retrieving effective parameters for metamaterials at oblique incidence," Phys. Rev. B 77(19), art. no. 195328, 1-8 (2008).

18. Heine, V., "On the general theory of surface states and scattering of electrons in solids," Proc. Phys. Soc. London 81(2), 300-310 (1963). 